\title{
Plantes utilisées dans le traitement traditionnel de l'infertilité féminine dans le département de Dabakala (Côte d'Ivoire)
}

\author{
KOMAN Silvère Romuald ${ }^{1}$, KPAN Wokapeu Blaise ${ }^{1,3 *}$, YAO Konan ${ }^{2,3,4}$, OUATTARA \\ Djakalia ${ }^{1,2}$ \\ ${ }^{1}$ Laboratoire de Botanique, UFR Biosciences, Université Félix HOUPHOUËT-BOIGNY, 22 BP 582 Abidjan 22 (Côte \\ d'Ivoire) \\ ${ }^{2}$ Centre National de Floristique (CNF), Abidjan, 01 BP 1303 Abidjan 03 (Côte d'Ivoire) \\ ${ }^{3}$ Centre Suisse de Recherches Scientifiques en Côte d'Ivoire (CSRS), 01 BP 1303 Abidjan 03 (Côte d'Ivoire) \\ 4Institut Botanique Aké-Assi d'Andokoi, BP 582 Abidjan 22 Abidjan 01, Côte d'Ivoire \\ *Auteur correspondant: kpanblaise@gmail.com,Cel: (+225) 78184098
}

Mots clés : plantes médicinales, matrone, infertilité féminine, Côte d'Ivoire

Keywords : medicinal plants, matrons, infertility of women, Côte d'Ivoire.

Publication date 31/10/2019, http://www.m.elewa.org/JAPS

\section{RESUME}

Le but de cette étude est de recenser et documenter les plantes médicinales utilisées traditionnellement contre l'infertilité féminine par les matrones (accoucheuses traditionnelles) dans le département de Dabakala (Côte d'Ivoire). Ainsi une enquête par questionnaire a été menée auprès de 71 matrones du département de Dabakala. Les résultats de cette enquête ont indiqué que 25 espèces reparties entre 23 genres et 18 familles sont utilisées par les matrones pour soigner divers cas d'infertilité. Les familles les plus représentées sont les Annonaceae et les Solanaceae. Parmi les espèces citées, Heliotropium indicum (Boraginaceae), Parkia biglobosa (Fabaceae), Xylopia aethiopica (Annonaceae), Vitellaria paradoxa (Sapotaceae) et Kigelia africana (Bignoniaceae) sont les plus recommandées. Les valeurs de VAUs de ces espèces varient de 0,42 à 0,24 . Ces résultats constituent une base de données pour les études ultérieures visant à évaluer les potentialités biologiques et chimiques de ces plantes.

\footnotetext{
ABSTRACT

The purpose of this study is to identify and document medicinal plants traditionally used by matrons (traditional midwives) against female infertility in the department of Dabakala (Côte d'Ivoire). A questionnaire survey therefore was conducted on 71 matrons in Dabakala department. The results of this survey showed that 25 species distributed among 23 types and 18 families are used by the matrones to treat various cases of infertility. The most represented families (groups of espece) are Annonaceae and Solanaceae. Among the mentioned species, Heliotropium indicum (Boraginaceae), Parkia biglobosa (Fabaceae), Xylopia aethiopica (Annonaceae), Vitellaria paradoxa (Sapotaceae) and Kigelia africana (Bignoniaceae) are the most recommended. The Use Agreement Value (VAU-valeur d'accord d'utilisation) (VAU) of these species vary from 0.42 to 0.24 . These results provide a database for subsequent studies to evaluate the biological and chemical potential of these plants.
} 


\section{INTRODUCTION}

L'infertilité est l'incapacité d'un couple à procréer ou à mener une grossesse à terme au bout d'une année après des rapports sexuels réguliers non protégés (José-Miller, 2007). Elle affecte 10 à 15\% des couples aux Etats-Unis (José-Miller, 2007) et 10 à $20 \%$ des couples en France (ORC and WHO, 2004). En Afrique, l'infertilité constitue un problème de santé publique (Nordeng et al., 2013). Elle affecte la vie des individus, des couples et des familles si bien qu'elle constitue un lourd fardeau pour leur développement socio-économique. En plus, dans nos sociétés africaines, l'enfant est indispensable et devient de ce fait la raison de la survie d'un couple. Ainsi, une femme sans enfant est considérée comme un déshonneur pour sa famille (Déléké Koko et al., 2009). Selon Adomou et al. (2012) le taux d'infertilité féminine est de $30 \%$ en Afrique subsaharienne L'infertilité peut-être primaire ou secondaire selon que la femme ait été ou non au moins une fois en grossesse (Ikechebelu et al., 2010). De nombreuses conditions peuvent lui être associées parmi lesquelles des facteurs non évitables (anatomique, problèmes génétiques, hormonaux et immunologiques) et évitables. Parmi ces derniers, on distingue les infections sexuellement transmissibles (IST), les infections après la parturition ou la chirurgie, la tuberculose du bassin et l'obésité (Larsen et al., 2007). La majorité des personnes infertiles vivent dans les pays en développement avec des taux les plus élevés du monde (30\%). Plusieurs traitements médicaux existent pour la stérilité. Ils incluent l'utilisation de médicaments de fertilité pour stimuler la «superovulation» qui correspond au développement et à la publication de plus d'un œuf par cycle ovulatoire. Aussi, existe-il des techniques entre

\section{METHODES D'ETUDE}

3.1 Site d'étude : Le milieu d'étude est le département de Dabakala en zone de savane soudanienne, au Centre-Nord de la Côte d'Ivoire (Figure 1). La ville de Dabakala est située entre $08^{\circ} 23^{\prime}$ de latitude Nord et $04^{\circ} 26^{\prime}$ de longitude Ouest, avec $258 \mathrm{~m}$ d'altitude. La autres, l'insémination intra-utérine et «hightech» et les traitements tels que les techniques de procréation assistée (ART) (Breart et De Mouzon, 1995). Mais ces traitements proposés par la médecine moderne sont coûteux et possèdent parfois de nombreux effets secondaires. De plus, les structures hospitalières capables d'offrir de tels traitements sont généralement très éloignées des zones rurales, où la plupart des femmes stériles est retrouvée. Ainsi, l'usage des plantes médicinales apparait aujourd'hui comme une alternative appropriée pour résoudre ce problème d'infertilité, qui est souvent cause de frustrations conjugales, de violences, de divorce ou de polygamie (Diame, 2010). Plusieurs études ont en effet démontré l'implication de nombreux métabolites secondaires issus des plantes médicinales dans la régulation des fonctions de reproduction (Binimbi-Massengo et al., 2007 ; Benalia A., 2016). En Côte d'Ivoire, en dépit des enquêtes ethnobotaniques réalisées sur les plantes médicinales (N’guessan et al., 2009, Béné et al., 2016 ; Ouattara et al., 2016), il est à noter que très peu d'études relatives au traitement spécifique de l'infertilité par les plantes, sont disponibles, d'où la nécessité de répertorier dans le but de les sauvegarder. C'est ainsi que ce travail a été initié sur les plantes médicinales utilisées dans le traitement traditionnel de l'infertilité féminine dans le Département de Dabakala (Côte d'Ivoire). Les objectifs de ce travail consistent à recenser et documenter les informations sur les plantes médicinales ainsi que les recettes médicamenteuses utilisées et proposées par les matrones pour le traitement traditionnel de l'infertilité féminine dans le département de Dabakala, en zone de savane soudanienne.

zone est à cheval sur le climat Baouléen (au Sud) et le climat soudanien (au Nord), avec une prédominance du climat soudanien. Ce dernier compte deux saisons caractérisées par le vent sec et poussiéreux de l'Harmattan. La zone d'étude couvre une superficie totale de 9671 
$\mathrm{km}^{2}$ et une densité de 20 habitants au $\mathrm{km}^{2}$. Il est limité au Nord par le District de Ferkessédougou, à l'Est par les Districts de Bouna et de Bondoukou et à l'Ouest par les Districts de Katiola et Niakara (Ouattara, 2007).

3.2 Échantillonnage : La présente étude a été réalisée à partir d'une série d'enquêtes ethnobotaniques utilisant un questionnaire préélaboré (Zerbo et al., 2007 ; Tra Bi et al., 2008 ; Fah et al., 2013). Le Département de Dabakala est composé de plus de 100 villages. Le choix des villages a été opéré à la suite d'une enquête préliminaire lors d'une mission exploratoire dans la zone d'étude.

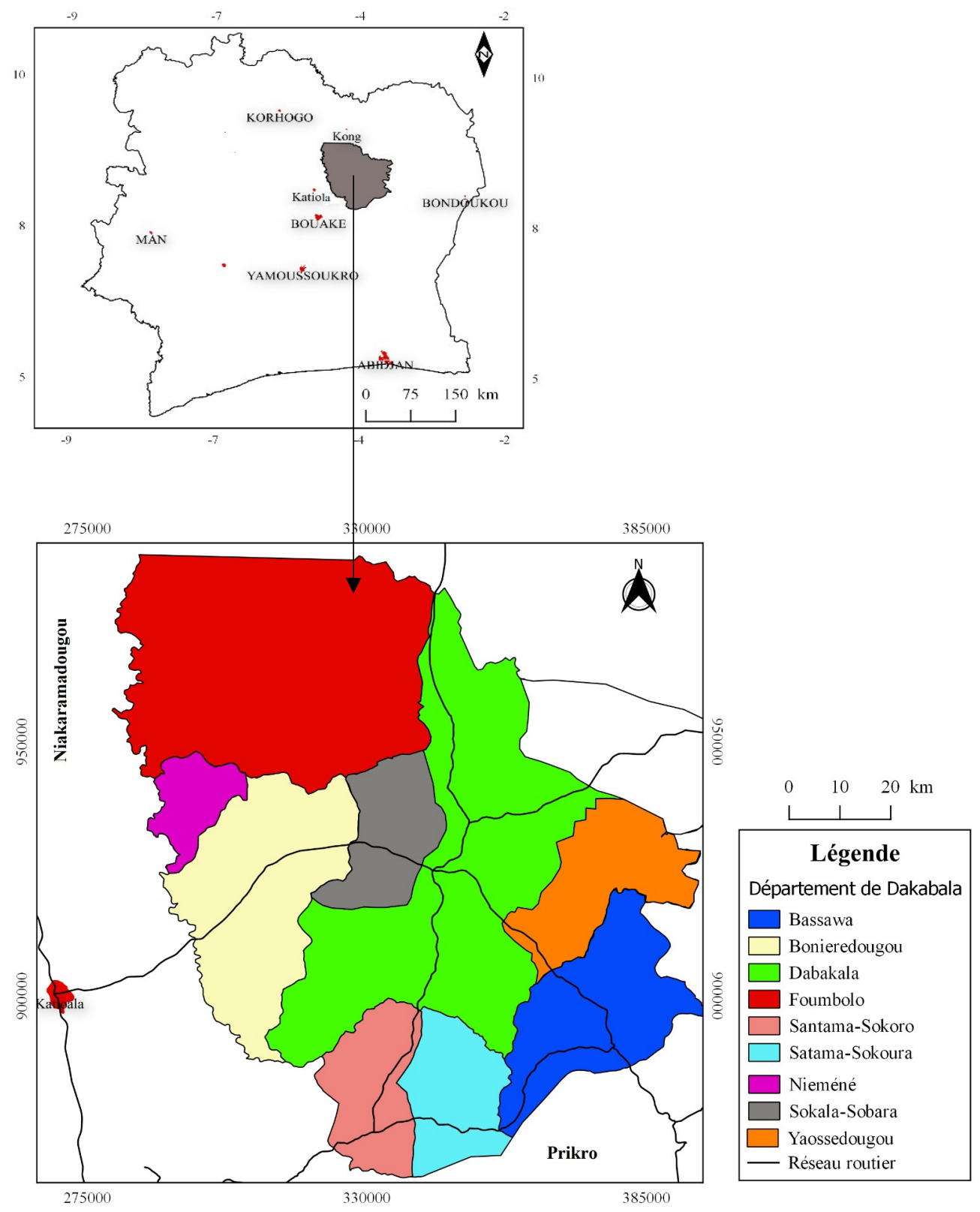

Figure 1 : Sous- préfectures échantillonnées et visitées dans le département de Dabakala 
Ainsi, les villages ont été sélectionnés d'une part suivant des transects allant du chef-lieu du du chef-lieu du département et orientés dans les quatre directions cardinales (Nord, Sud, Est, Ouest) selon 4 axes routiers. D'autre part, nous avons procédé à un échantillonnage non probabiliste dit sélection orientée comme méthode d'échantillonnage. Les villages ont été sélectionnés à cet effet, sur la base de leur accessibilité, la réputation des matrones ou accoucheuses traditionnelles afin d'obtenir des informations assez représentatives de la zone d'étude. Ainsi, par cette méthode, nous avons retenu 60 localités dans le département de Dabakala (Figure 2).

\subsection{Matrones rencontrées : 71}

accoucheuses traditionnelles (matrones) ont été enquêtées. Pour y parvenir, nous nous sommes conformés aux pratiques locales. En effet, avant l'entretien, une bouteille de boisson alcoolisée ou une somme d'argent moyennant le service rendu a été offerte à notre interlocutrice. Après l'entretien et avant de passer à la récolte des échantillons, une somme équivalente au prix local d'un poulet de couleur noire ou souvent une boisson alcoolisée a été également offerte. Ce geste symbolisant l'autorisation ou le droit d'entrer dans la forêt ou la savane est obligatoire. Les questions ont été posées dans la langue locale appelée le Djimini. Nous nous sommes fait accompagner de guidesinterprètes.

3.4 Traitement des données : A partir des variables échantillonnées, notamment le profil de chaque enquêté (sexe, âge, niveau d'études, ethnie) nous avons caractérisé les matrones ou accoucheuses traditionnelles de la zone d'étude. Les données ont été enregistrées sur un tableur Excel, puis analysées avec les logiciels Excel 2010, Epidata et SPSS 20.0. Le test de Khi-2 (égalité des distributions) a été utilisé en vue de comparer les pourcentages des parties utilisées, le mode de préparation et le mode d'administration et a permis de vérifier si les différences sont significatives. 

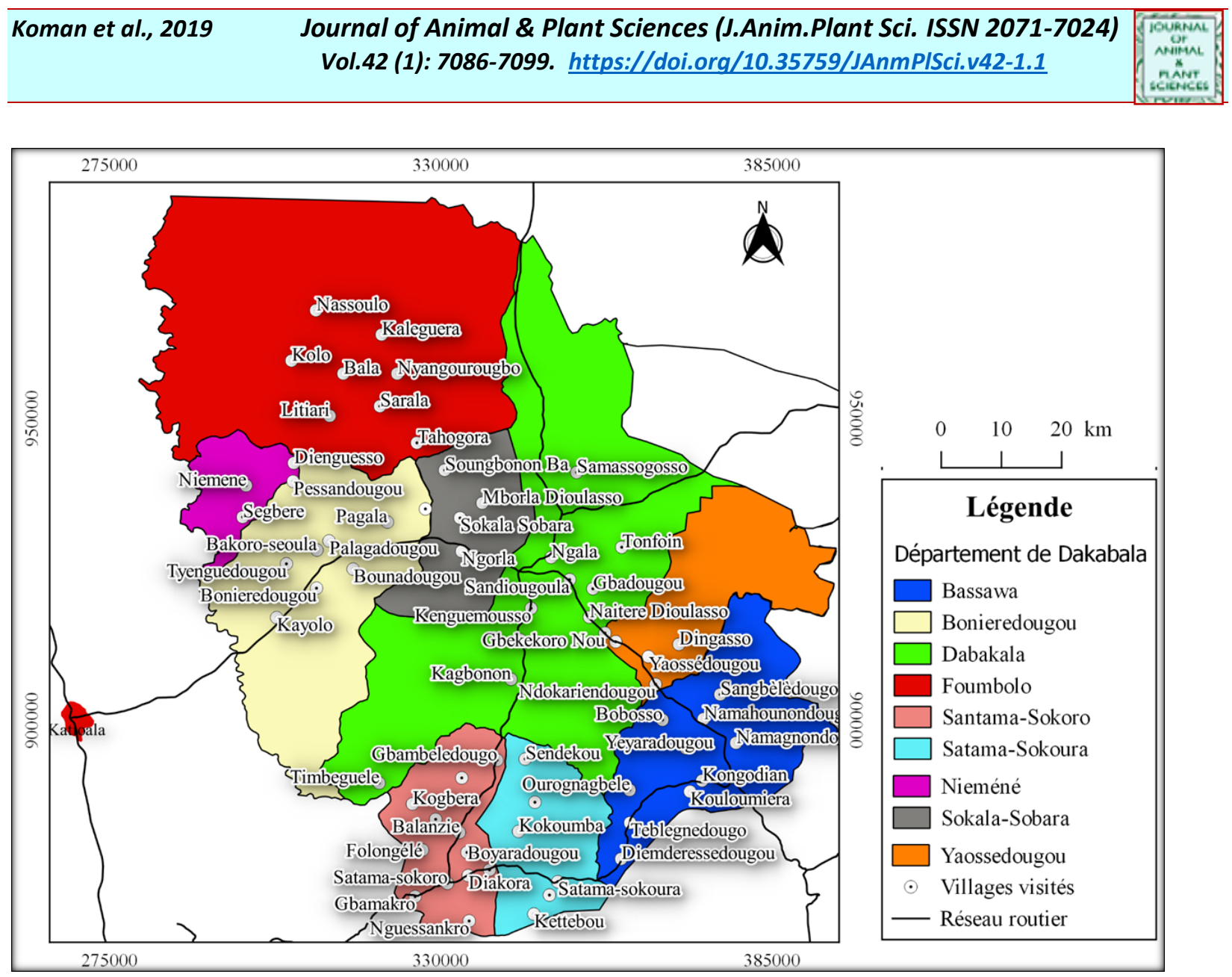

Figure 2 : Localités visitées dans le département de Dabakala

Pour mieux interpréter la valeur culturelle médicinale des plantes, nous avons utilisé l'indice de la Valeur d'Accord d'Utilisation (VAUs) (Ilumbe, 2010), qui combine l'indice de valeur d'utilisation (VUs) (Philips et al., 1993) et l'indice de confirmation proposé par Byg et Balslev (2001). Cet indice se présente comme suit :

VAUs = VUs $\mathrm{x}$ ICs (1)

L'indice de la valeur d'utilisation VUs s'exprime par:

VUs $=\sum_{\mathrm{i}=1}^{\mathrm{n}} \mathrm{U}_{\mathrm{is}} / \mathrm{ns}(2)$

où Uis indique le nombre d'utilisations de l'espèce $s$ mentionné par l'informateur i et ns correspond au nombre de personnes ayant cité

\section{RESULTATS}

4.1 Étude botanique: Au total, nous avons identifié 25 plantes médicinales (Tableau 2) réparties en 23 genres et 18 familles. Ces cette espèce. Cette technique permet d'évaluer l'importance culturelle des plantes (Trotter et Logan, 1986 ; Thomas et al., 2009). Pour la présente étude, nous avons utilisé la formule d'origine proposée par Byg et Balslev (2001) pour exprimer le consensus d'informateurs ou l'indice de confirmation des plantes médicinale. Ainsi, le consensus d'informateurs se calcule comme suit :

$\mathrm{IC}_{\mathrm{s}}=\mathrm{Na} / \mathrm{Nt}(3)$

où ICs est l'indice de confirmation,

$\mathrm{Na}=$ nombre de personnes ayant cité cette espèce et $\mathrm{Nt}=$ nombre total de personnes interviewées.

espèces interviennent dans la préparation de 33 recettes médicamenteuses dont 8 sont des associations de 2 plantes. 
Tableau 2 : Nombre de genres et d'espèces par famille utilisées contre l'infertilité féminine

\begin{tabular}{l|c|c}
\hline Familles & Nombres d'espèces & Nombre de genres \\
\hline Annonaceae & 3 & 3 \\
\hline Solanaceae & 3 & 2 \\
\hline Euphorbiaceae & 2 & 1 \\
\hline Fabaceae & 2 & 2 \\
\hline Asteraceae & 2 & 2 \\
\hline *Autres & 1 & 1 \\
\hline
\end{tabular}

*Papaveraceae, Boraginaceae, Amaranthaceae, Zingiberaceae,

Plantaginaceae, Bignoniaceae, Phyllanthaceae, Sapotaceae, Piperaceae, Portulacaceae, Sapindaceae, Combretaceae, Verbenaceae

Les plantes recensées sont composées de 6 arbres, 4 arbustes, 2 arbrisseaux, 12 herbes et 1 liane (Tableau 3).

Tableau 3 : Répartition des différentes formes biologiques des plantes utilisées contre l'infertilité féminine

\begin{tabular}{l|c|c|c|c}
\hline \multirow{2}{*}{ Types morphologiques } & \multicolumn{2}{|c|}{ Nombre d'espèces } & \multicolumn{2}{c}{ Nombre de citations } \\
\cline { 2 - 5 } & Djimini & Djamala & Djimini & Djamala \\
\hline Arbre & 6 & 4 & 45 & 40 \\
\hline Arbrisseau & 2 & 1 & 21 & 25 \\
\hline Arbuste & 4 & 2 & 26 & 30 \\
\hline Herbe & 12 & 7 & 75 & 80 \\
\hline Liane & 1 & 1 & 9 & 10 \\
\hline
\end{tabular}

4.2 Étude ethnobotanique: L'enquête ethnobotanique a permis d'interviewer 71 matrones dans le département de Dabakala. La quasi-totalité des matrones rencontrées sont animistes $92 \%$ et appartient au groupe ethnique Djimini (75\%). La plupart des matrones interrogées ont plus vingt ans d'expérience
$(47,9 \%)$. Concernant le niveau d'instruction des matrones du département de Dabakala, les résultats montrent que $74.6 \%$ ne sont pas scolarisées. Les adultes dont l'âge varie de 55 à 65 ans constituent la frange d'âge la plus impliquée dans cette activité, avec 73, $2 \%$ des personnes interrogées (Tableau 4). 
Tableau 4 : Caractéristiques sociodémographiques des matrones dans le département de Dabakala $(\mathrm{n}=71)$.

\begin{tabular}{|c|c|c|}
\hline Paramètres & Effectifs & Pourcentage \\
\hline \multicolumn{3}{|l|}{ Ethnie } \\
\hline Djamala & 18 & 25 \\
\hline Djimini & 53 & 75 \\
\hline \multicolumn{3}{|l|}{ Année d'expérience } \\
\hline 10 & 7 & 9,9 \\
\hline 15 & 9 & 12,7 \\
\hline 20 & 34 & 47,9 \\
\hline 25 & 11 & 15,5 \\
\hline 30 & 10 & 14,1 \\
\hline \multicolumn{3}{|l|}{ Niveau d'instruction } \\
\hline lettré & 18 & 25,4 \\
\hline Illettré & 53 & 74,6 \\
\hline \multicolumn{3}{|l|}{ Classe d'âges } \\
\hline$\leq 55$ & 11 & 15,5 \\
\hline ] 55-65] & 52 & 73,2 \\
\hline$] 65 ;+[$ & 8 & 11,3 \\
\hline
\end{tabular}

Concernant les parties de plantes utilisées, six types d'organes sont utilisés pour le traitement de l'infertilité féminine (Tableau 5). En plus de ces différents organes, la plante entière de certaines herbes est aussi utilisée. Les feuilles $(35,42 \%)$, les fruits $(19,58 \%)$ et les écorces de racines $(19,17 \%)$ sont les organes les plus sollicités.

Tableau 5 : Proportions des organes de plantes contre l'infertilité féminine

\begin{tabular}{l|c|c}
\hline Partie utilisée & Effectifs & Proportions(\%) \\
\hline Feuilles & 85 & 35,42 \\
\hline Fruits & 47 & 19,58 \\
\hline Ecorces de racines & 46 & 19,17 \\
\hline Ecorces de tiges & 35 & 14,58 \\
\hline Plante entière & 19 & 7,92 \\
\hline Graine & 5 & 2,08 \\
\hline Rhizome & 3 & 1,25 \\
\hline
\end{tabular}

Khi-2 = 168,14; $\mathrm{dl}=6 ; \mathrm{P}<0,001$

Ainsi, Pour faciliter l'administration des drogues, huit techniques de préparation sont employées à savoir : la décoction, le pétrissage, la macération, la calcination, la mastication, la trituration, l'infusion et l'expression. Parmi eux, le décocté est la forme médicamenteuse la plus utilisée (Tableau 6). Les différentes voies administrations (Tableau 6) utilisées dans le cas d'infertilité féminin dans le département de Dabakala sont la voie orale $(53 \%)$, la voie cutanée $(35 \%)$ et la voie anale $(12 \%)$. 
Tableau 6 : Répartition des différentes techniques de préparation et d'administration de plantes utilisées contre l'infertili

\begin{tabular}{|c|c|c|c|c|}
\hline $\mathbf{N}^{\circ}$ & Noms scientifiques & Organe utilisé & Mode de préparation & Mode d'adminis \\
\hline 1 & Heliotropium indicum & Feuilles & $\begin{array}{l}\text { Décoction/pétrissage/ } \\
\text { Trituration/expression }\end{array}$ & Voie anale/voie c \\
\hline 2 & Parkia biglobosa & Ecorces de tiges/écorces de racines & Décoction/macération & Voie orale/voie a \\
\hline 3 & Xylopia aethiopica & Fruits & Décoction/macération/calcination & Voie orale/voie v \\
\hline 4 & Vitellaria paradoxa & $\begin{array}{l}\text { Feuilles/écorces de tiges/écorces de } \\
\text { racines }\end{array}$ & Décoction/mastication/pétrissage & $\begin{array}{l}\text { Voie orale/voie a } \\
\text { voie nasale/voie }\end{array}$ \\
\hline 5 & Kigelia africana & Feuilles/écorces de tiges & $\begin{array}{l}\text { Décoction/calcination/ } \\
\text { Trituration/pétrissage }\end{array}$ & Voie orale/voie c \\
\hline 6 & Annona senegalensis & $\begin{array}{l}\text { Feuilles/écorces de tiges/écorces de } \\
\text { racines }\end{array}$ & Décoction & Voie orale/voie c \\
\hline 7 & Scoparia dulcis & Feuilles & Décoction/pétrissage/expression & $\begin{array}{l}\text { Voie orale/voie a } \\
\text { nasale/voie cutan }\end{array}$ \\
\hline 8 & Jatropha gossypiifolila & Feuilles/ racines & Décoction/trituration/macération & Voie orale/voie a \\
\hline 9 & Argemone mexicana & Feuilles/ plantes entières & Décoction/pétrissage & Voie orale/voie a \\
\hline 10 & Capsicum frutescens & Fruits & Pétrissage/Trituration & Voie anale \\
\hline 11 & Ageratum conyzoides & Feuilles & Décoction/ Macéré+miel & Voie orale/voie c \\
\hline 12 & Alternanthera pungens & Feuilles/ plantes entières & Pétrissage & Voie anale \\
\hline 13 & Jatropha curcas & $\begin{array}{l}\text { Feuilles/écorces de tiges/écorces de } \\
\text { racines }\end{array}$ & Décoction/macération & Voie orale \\
\hline 14 & Piliostigma thonningii & Feuilles/écorces de tiges/fruits & Décoction/mastication & Voie orale \\
\hline 15 & Zingiber officinale & Rhizomes & Expression/pétrissage & Voie cutanée \\
\hline 16 & Piper guineense & Graines & $\begin{array}{l}\text { Décoction/Macération/ } \\
\text { Calcination/pétrissage }\end{array}$ & Voie anale \\
\hline 17 & Portulaca oleracea & Feuilles/ plantes entières & Pétrissage/trituration/ expression & Voie anale \\
\hline 18 & Aspilia bussei & Feuillles & Décoction & Voie orale \\
\hline 19 & Blighia sapida & Feuillles & Décoction & Voie orale \\
\hline 20 & Capsicum annum & Fruits & Pétrissage/trituration & Voie anale \\
\hline 21 & Combretum glutinosum & Feuilles & Décoction & Voie orale \\
\hline 22 & Combretum micranthum & Feuilles & Décoction/pétrissage/expression & Voie orale/ voie \\
\hline 23 & Nicotiana tabacum & Feuilles & Décoction & Voie orale/ voie \\
\hline 24 & Phyllanthus amarus & Plante entière & Décoction/pétrissage/trituration & Voie orale \\
\hline 25 & Uvaria tortilis & Feuilles/écorces de tiges & Décoction & Voie orale \\
\hline
\end{tabular}


Si les valeurs de l'indice VUs mettent en première position les espèces Heliotropium indicum, Scoparia dulcis, Zingiber officinale, Parkia biglobosa, Jatropha gossypiifolila avec des valeurs supérieures ou égale à 1,11 , celui basé sur des valeurs de ICs met en première position les espèces Heliotropium indicum, Parkia biglobosa, Xylopia aethiopica, Vitellaria paradoxa, Kigelia africana avec des valeurs supérieures ou égales à 0 , 24. Les valeurs des indices VUs et ICs corrèlent positivement et significativement avec les valeurs de VAUs (coefficient de corrélation de Spearman respective égale à 0,84 et 0,28 pour $\mathrm{p}<0,001)$. En associant l'indice VUs et ICs sur l'ensemble des espèces végétales intervenant dans le traitement de l'infertilité féminine dans le Département de Dabakala
(Tableau 7), ce sont les espèces Heliotropium indicum, Parkia biglobosa, Xylopia aethiopica, Vitellaria paradoxa, Kigelia africana, Annona senegalensis, Scoparia dulcis, Jatropha gossypiifolila, Argemone mexicana, Capsicum frutescens qui ont chacune un VAUs élevée. Ces espèces peuvent être intéressantes dans le traitement de cette affection. Aussi, la confrontation de la liste générale des espèces avec celle de 1'UICN (2015) et celle de Aké-Assi (1998) nous a permis d'identifier 02 espèces végétales à statut particulier. On note d'une part, 1 espèce vulnérable (VU) figurant sur la liste rouge de l'UICN, Vitellaria paradoxa. D'autre part, 1 espèce qui se retrouve sur la liste de Aké-Assi comme espèces rares, devenues rares et en voie d'extinction. Il s'agit de Uvaria tortilis.

Tableau 7 : Liste des plantes utilisées contre infertilité féminine et leur valeur d'accord d'utilisation (VAUs).

\begin{tabular}{|c|c|c|c|c|c|c|c|c|c|}
\hline $\mathbf{N}$ & $\begin{array}{l}\text { Noms } \\
\text { scientifiques }\end{array}$ & Familles & Noms vernaculaires & NR & $\begin{array}{c}\mathbf{N} \\
\mathbf{I}\end{array}$ & $\mathrm{N}$ & VU & ICs & $\begin{array}{c}\text { VAU } \\
\text { s }\end{array}$ \\
\hline 1 & Heliotropium indicum & Boraginaceae & Tchegorlornifoulé & 1 & 23 & 3 & 1,34 & 0,32 & 0,42 \\
\hline 2 & Parkia biglobosa & Fabaceae & Nindigué & 1 & 22 & 2 & 1,13 & 0,31 & 0,35 \\
\hline 3 & Xylopia aethiopica & Annonaceae & Kanifi & 2 & 22 & 2 & 1,00 & 0,31 & 0.31 \\
\hline & & & & & & 2 & & & \\
\hline 4 & Vitellaria paradoxa & Sapotaceae & Letigué & 1 & 20 & 2 & 1,00 & 0,28 & 0,28 \\
\hline 5 & $\begin{array}{llll}V^{*} & l^{\circ} & f^{\prime}\end{array}$ & & & 1 & 17 & 0 & 100 & & 020 \\
\hline 5 & Kigelia africana & Bignoniaceae & Sidjan & 1 & 17 & $\begin{array}{l}1 \\
7\end{array}$ & 1,00 & 0,24 & 0,24 \\
\hline 6 & Annona senegalensis & Annonaceae & Lofigué & 1 & 10 & 1 & 1,00 & 0,14 & 0,14 \\
\hline 7 & Scoparia dulcis & Plantaginaceae & Gaigniwoué & 2 & 10 & 1 & 1,20 & 0,17 & 0,14 \\
\hline & & & & & & 2 & & & \\
\hline 8 & Jatropha gossypiifolila & Euphorbiaceae & Ploplo & 1 & 9 & 1 & 1,11 & 0,13 & 0,14 \\
\hline & & & & & & 0 & & & \\
\hline 9 & Argemone mexicana & Papaveraceae & Drassifi & 1 & 5 & 5 & 1,00 & 0,14 & 0,14 \\
\hline 10 & Capsicum frutescens & Solanaceae & Gbéhé & 2 & 5 & 5 & 1,00 & 0,14 & 0,14 \\
\hline 11 & Ageratum conyzoides & Asteraceae & $\begin{array}{l}\text { Moussocôrôni-cougê } \\
\text { (Malinké) }\end{array}$ & 2 & 4 & 4 & 1,00 & 0,06 & 0,06 \\
\hline 12 & $\begin{array}{l}\text { Alternanthera } \\
\text { pungens }\end{array}$ & $\begin{array}{l}\text { Amaranthacea } \\
\text { e }\end{array}$ & Kambessaba & 1 & 4 & 4 & 1,00 & 0,06 & 0,06 \\
\hline 13 & Jatropha curcas & Euphorbiaceae & Kapara & 1 & 4 & 4 & 1,00 & 0,06 & 0,06 \\
\hline 14 & Zingiber officinale & Zingiberaceae & Tragbéhé & 2 & 3 & 4 & 1,33 & 0,04 & 0,06 \\
\hline 15 & Piliostigma thonningii & Fabaceae & Yewagnan & 1 & 3 & 3 & 1,00 & 0,04 & 0,04 \\
\hline 16 & Piper guineense & Piperaceae & Fêfê (Malinké) & 2 & 2 & 2 & 1,00 & 0,03 & 0,03 \\
\hline
\end{tabular}




\begin{tabular}{l|l|l|l|l|l|l|l|l|l}
\hline $\mathbf{1 7}$ & Portulaca oleracea & Portulacaceae & Gbigbinin, Nandini & 1 & 2 & 2 & 1,00 & 0,03 & 0,03 \\
\hline $\mathbf{1 8}$ & Aspilia bussei & Asteraceae & Deniwor & 1 & 1 & 1 & 1,00 & 0,01 & 0,01 \\
\hline $\mathbf{1 9}$ & Blighia sapida & Sapindaceae & Gôkougô & 1 & 1 & 1 & 1,00 & 0,01 & 0,01 \\
\hline $\mathbf{2 0}$ & Capsicum annum & Solanaceae & Gbéhé & 1 & 1 & 1 & 1,00 & 0,01 & 0,01 \\
\hline $\mathbf{2 1}$ & $\begin{array}{l}\text { Combretum } \\
\text { glutinosum }\end{array}$ & Combretaceae & Wobihin & 2 & 1 & 1 & 1,00 & 0,01 & 0,01 \\
\hline $\mathbf{2 2}$ & Lippia multiflora & Verbenaceae & N'golobè (Malinké) & 1 & 1 & 1 & 1,00 & 0,01 & 0,01 \\
\hline $\mathbf{2 3}$ & Nicotiana tabacum & Solanaceae & Gbossoro (Malinké) & 1 & 1 & 1 & 1,00 & 0,01 & 0,01 \\
\hline $\mathbf{2 4}$ & Phyllanthus amarus & Phyllanthaceae & Sougnassi (Malinké) & 1 & 1 & 1 & 1,00 & 0,01 & 0,01 \\
\hline $\mathbf{2 5}$ & Uvaria tortilis & Annonaceae & Pedjan & 2 & 1 & 1 & 1,00 & 0,01 & 0,01 \\
\hline
\end{tabular}

$\mathbf{N} . \mathbf{R}=$ nombre de recettes, $\mathbf{N} . \mathbf{I}=$ nombre d'informateurs, $\mathbf{N} . \mathbf{C}=$ nombre de citations, VUs = valeur d'utilisation, ICs $=$ indice de confirmation

\section{$5 \quad$ DISCUSSION}

Cette étude a été réalisée auprès de 71 matrones à travers une série d'enquêtes ethnobotaniques. Le choix de celles-ci pourrait se justifier par le fait qu'elles sont les pionnières du savoir ancestral depuis le début de la grossesse jusqu'à la puerpéralité. Pourchez (2011) a également montré qu'elles ont longtemps occupé le principal rôle dans la surveillance et la protection de la fécondité, de l'accouchement et de la première partie du cycle de vie des nouveaux nés.. Aussi, elle constitue une maladie fréquemment traitée par les matrones de cette zone. Adomou et al. (2012) ont également montré dans leurs études que l'infertilité féminine était la 3ème maladie fréquemment traitée par les vendeuses phytothérapeutes du Bénin. La majorité des matrones interrogées (73\%) ont un âge qui varie de 55 à 65 ans et au moins vingt année d'expériences. L'âge et le nombre élevé d'années d'expériences pourraient traduire la fiabilité de leurs recettes. En effet, la connaissance des usages des plantes médicinales et leurs propriétés, est généralement acquise suite à une longue expérience accumulée et transmise d'une génération à l'autre (Benlamdini et al., 2014). Les plantes médicinales intervenant dans le traitement de l'infertilité féminine sont diversement utilisées. Certaines recettes comprennent un organe ou une combinaison d'organes tels que les racines, les écorces, les fruits, les feuilles, etc. Les feuilles (y compris les rameaux feuillés) sont les parties les plus utilisées avec 35\%. Cette prédominance pourrait s'expliquer par le fait que les feuilles sont par excellence le siège de synthèse des métabolites secondaires de la plante. Par conséquent, elles contiennent de nombreux groupes chimiques responsables des vertus et propriétés biologiques de la plante (Mangambuet al., 2014). Les études ethnobotaniques menées par Ambé et al. (2015), Kipré et al. (2017) ont également rapporté que les feuilles sont les organes végétaux les plus utilisés en médecine traditionnelle dans le traitement de diverses affections. Cette forte utilisation des feuilles ne constitue pas une menace pour la plante d'après les travaux de Ouattara (2006). Car selon cet auteur, prélever jusqu'à $50 \%$ des feuilles d'une plante ne l'affecte pas de manière considérable. Aussi, l'utilisation des fruits en deuxième position par les matrones $(19,58 \%)$ est due au fait qu'elle serait sans incidence et moins préjudiciable pour la plante que les racines et les écorces. En effet, la récolte des racines d'un arbre et/ou de son écorce semble avoir plus d'incidences écologiques néfastes que la récolte des feuilles (Cunningham, 2001). Par conséquent, leur prélèvement supprime la possibilité de ravitaillement de la plante en éléments nutritifs, ce qui affecte son aspect végétatif ainsi que sa physiologie (Yapi, 2013). Néanmoins, l'impact d'une utilisation particulière sur un arbre, dépend de la partie utilisée et de la méthode de prélèvement. S'agissant des organes utilisés dans les recettes, il est à remarquer qu'ils n'ont pas la même importance. Certains organes, surtout les fruits et les graines, sont utilisés 
comme des ingrédients auxiliaires ou adjuvants, permettant de renforcer l'action thérapeutique des composantes principales des recettes et de traiter les symptômes secondaires de la maladie (Joy et al., 2001) ; c'est le cas des fruits ou graines de Vitellaria paradoxa et Parkia biglobosa. Ces derniers ont été cités parmi les espèces les plus connues dans le traitement de l'infertilité féminine. Yao (2010) a déjà cité Parkia biglobosa avec plus de $90 \%$ de connaissance parmi les fruits sauvages les plus utilisés par le groupe ethnique Malinké de Côte d'Ivoire. Le soumbala serait un régulateur de la tension artérielle. Ceci explique sa grande consommation par l'ensemble des populations de la Côte d'Ivoire. En Afrique de l'ouest, des études ont également montré que Parkia biglobosa et Vitellaria paradoxa sont utilisés en décoction par les phytothérapeutes contre la diarrhée (Nikiema, 2010). La décoction et le pétrissage sont les modes préparations les plus citées. Cela pourrait se justifier par le fait que la décoction permet de recueillir le plus de principes actifs et atténue ou annule l'effet toxique de certaines recettes (Salhi et al., 2010). Plusieurs auteurs ont observé dans leurs travaux la forte utilisation du décocté dans le traitement de plusieurs affections en médecine traditionnelle (Gnagne et al., 2017). Aussi, l'état et le mode de conservation dans lequel se trouve l'organe de la plante peut conditionner l'efficacité ou le mode d'action du médicament traditionnel. En effet, les formes médicamenteuses obtenues à partir des organes de plantes fraiches sont consommées rapidement car elles ne permettent pas une longue conservation que les organes secs. La

\section{CONCLUSION}

Les enquêtes réalisées auprès des matrones des différentes localités du département de Dabakala ont permis de recenser 32 recettes médicinales. Elles sont préparées à partir de 25 espèces différentes de plantes médicinales et utilisées, souvent seules, mais surtout en association à d'autres espèces de plantes. Heliotropium indicum, Parkia biglobosa, Xylopia aethiopica, Vitellaria paradoxa, Kigelia africana, durée de conservation ne dépasse guère cinq jours. Après ce délai, le médicament devient impropre à la consommation, inefficace voire toxique à cause de la dégradation des molécules chimiques alors que les plantes à l'état sec se conservent plus longtemps (Dibong et al., 2011). Les préparations citées sont pratiquement toutes prescrites par voie orale et voie anale. La prescription par voie orale pourrait s'expliquer par le fait que la maladie est liée à des infections bactériennes, fongiques et/ou parasitaires localisées dans des organes profonds. Pour les atteindre, le composé doit transiter par l'appareil digestif pour en faciliter l'assimilation et l'action (Tra Bi et al., 2008). Aussi, elle pourrait se justifier par le fait qu'il peut avoir des risques d'atteintes viscérales dans certains cas. Nonobstant le caractère secret de leur fonction, la réciprocité d'emplois des plantes utilisées contre l'infertilité féminine entre les matrones, démontrent que ces dernières arrivent aussi à réaliser des échanges de connaissances entre elles. Nous pouvons croire qu'elles vendent aussi leurs connaissances s'il y a nécessité. Ces échanges ne se font pas seulement entre les spécialistes d'une même communauté, on assiste à des cas où un djimini transmet un secret à un djamala. Le contraire n'est pas aussi impossible. Toutefois, cette situation témoigne de la complexité du domaine de la médecine et de la pharmacopée traditionnelle (Zerbo, 2007). En effet, les recettes et la connaissance des plantes qui entrent dans leur composition constituent des secrets qui ne sont confiés à une tierce personne que quand le dépositaire le veut (Ilumbe, 2010)

Annona senegalensis sont les plantes les plus sollicitées dans le traitement de l'infertilité féminine dans le département de Dabakala avec un VAUs élevé qui varie de 0,42 à 0,24. Ces différentes plantes et recettes ont des spécificités d'action et sont plus indiquées dans le traitement d'une forme particulière de cause de stérilité. Selon la bibliographie que nous avons effectuée plusieurs parmi les plantes 
recensées n'ont pas encore fait l'objet d'études de laboratoire pour la vérification de leurs effets bénéfiques sur la fertilité féminine, et pourraient constituer des sujets intéressants de recherche. Aussi, le criblage phytochimique au

\section{REMERCIEMENTS}

Nos remerciements s'adressent aux chefs de village, aux conseillers communautaires, aux Agents de Santé communautaires (ASC) ainsi qu'aux matrones pour leur accessibilité, leur disponibilité et leur franche collaboration à cette étude. Nous remercions également l'ONG CASES (Centre d'Animation Sanitaire et d'études Sociales) et Heath Alliance laboratoire est nécessaire pour connaître les composés chimiques contenus dans les principales plantes utilisées et leur degré de toxicité.

International (HAI) qui ont été favorable à l'exécution de cette étude. Nous remercions, enfin, Monsieur ASSI Yapo Jean, Technicien botaniste au Centre National de Floristique, de l'Université Félix HOUPHOUET-BOIGNY pour sa contribution à l'identification des plantes recensées.

\section{REFERENCES BIBLIOGRAPHIQUES}

Adjanohoun E. et Aké-Assi L: 1979. Contribution au recensement des plantes médicinales de Côte d'Ivoire. Centre National de Floristique, Université d'Abidjan, 358 p.

Adomou AC, Yedomonhan H, Djossa B, Legba SI, Oumorou M. et Akoegninou A : 2012. Etude ethnobotanique des plantes médicinales vendues dans le marché d'Abomey- Calavi au Bénin. International Journal of Bioliogical and Cheminical Scences., 6(2): 745-772.

Ambe ASA, Ouattara D, Tiebre M-S, Vroh Bi TA, Zirihi GN. et N'guessan KE: 2015.-Diversité des plantes médicinales utilisées dans le traitement traditionnel de la diarrhée sur les marchés d'Abidjan (Côte d'Ivoire). Journal of Animal \& Plant Sciences, 26 (2): 4081-4096.

Aké-Assi L: 2011. Abrégé de médecine et pharmacopée africaines : Quelques plantes employées traditionnellement dans la couverture des soins de santé primaire. NEI-CEDA, Abidjan, 157 p.

Benlamdini N, Elhafian M, Atmane R. et Lahcen Z: 2014. Étude floristique et ethnobotanique de la flore médicinale du Haut Atlas oriental (Haute Moulouya), Maroc. Journal of Applied Biosciences, 78: 6771- 6787
Benalia A : 2016. Effet de l'exposition chronique aux alcaloïdes totaux $\mathrm{du}$ tabac sur la fonction reproductrice chez les rats mâles wistar: effet preventif de la fraction d'acetate d'ethyle de l'extrait de the vert. Thèse de Doctorat de l'Université Djillali Liabes de Sidi BelAbbés, Djillali Liabes, Algerie, 170 p.

Binimbi-Massengo A, N'Zongi J, Abena AA. et Diatewa M: 2007. Inventaire des plantes médicinales utilisées dans le traitement des menaces d'avortement à Brazzaville. Revue Méd. Pharm. Afr., 20: 155-164.

Breart G. et De Mouzon J : 1995. AMP vigilance. Bulletin de l'Académie Nationale de Médecine 179, 1759-1764.

Byg A. et Balslev H : 2001. Diversity and use of palms in Zahamena, eastern Madagascar. Biodiversity and Conservation, 10: 951-970.

Déléké Koko IKE, Djego J, Gbénou J, Hounzangbe-Adoté SM. et Brice Sinsin: 2009. Etude ethnobotanique des plantes galactogènes et emménagogues utilisées dans les terroirs riverains à la Zone Cynégétique de la Pendjari. International Journal of Biological and Chemical Sciences, 3(6): 1226-1237. 
Cunningham AB : 2002. Applied ethnobotany: People, wild plant use and conservation. People and Conservation Manuel, Earth scan publications Ltd, 300 p.

Devendrakumar D. et Anbazhagan M : 2012. Ethnoveterinary medicinal plants used in Perambalur District, Tamil Nadu. Research in Plant Biology, 2 (3): 24-30.

Diame GLO : 2010. Ethnobotany and Ecological Studies of Plants used for Reproductive Health: A Case Study at Bia Biosphere Reserve in the Western region of Ghana. Accra, Ghana,

Dibong SD, Mpondo ME, Ngoye A, Kwin MF. et Betti JL: 2011. Ethnobotanique et phytomédecine des plantes médicinales de Douala, Cameroun. Journal of Applied Biosciences, 37: 2496 - 2507.

Elspeth H, James R, Peter F, Mark WC. et David JH: 2009. The Linear Angiosperm Phylogeny Group (LAPG) III: a linear sequence of the families in APG III. Botanical Journal of the Linnean Society, 161: 128-131.

Fah L, Klotoé JR, Dougnon V, Koudokpon H, Fanou VBA, Dandjesso C.et Loko F : 2013. Étude ethnobotanique des plantes utilisées dans le traitement du diabète chez les femmes enceintes à Cotonou et Abomey-Calavi (Bénin). Journal of Animal \&Plant Sciences. Vol.18, Issue 1: 2647-2658.

Gnagne AS, Camara D, Fofié NBY, Béné K. et Zirihi G : 2017. Étude ethnobotanique des plantes médicinales utilisées dans le traitement du diabète dans le Département de Zouénoula (Côte d'Ivoire). Journal of Applied Biosciences

Houmenou V, Adjatin A, Tossou M G, Yedomonhan H, Dansi A, Gbenou J. et

Akoegninou: 2017. Etude ethnobotanique des plantes utilisées dans le traitement de la stérilité féminine dans les départements de l'Ouémé et du plateau au Sud Bénin. International Journal of Biological and Chemical Sciences, 11(4): 1851-1871.

Ikechebelu JI, Eke NO, Eleje GU. et Umeobika JC : 2010. Comparism of the diagnostic accuracy of Laparoscopy with dye test and Hysterosalpingography in the evaluation of Infertile women in Nnewi, Nigeria. Tropical Journal of Laparo Endoscopy, 1(1): 39-44

Ilumbe G: 2010. Utilisation des plantes en médecine traditionnelle par les Pygmées (Ba-twa) et les Bantous (Ba-Oto) du territoire de Bikoro, province de l'Equateur en RD Congo. Thèse de Doctorat à l'Université Libre de Bruxelles (Belgique), 237 p.

Jose-miller AB, Boyden JW. et Frey KA : 2007. Infertility. Am Fam Physician, 75(6): 849-856.

Kipre GR, Offoumou MR, Silue KD, Bouabre GM, Zirihi GN et Djaman AJ : 2017. Enquête ethnopharmacologique des plantes antipaludiques dans le département d'Agboville, Sud-Est de la Cote d'Ivoire. Journal of Applied Biosciences, 109: 10618-10629.

Larsen SH, Wagner G. et Heitmann BL : 2007. Sexual function and obesity. International Journal of Obesity 31, 1189-1198.

Mangambu M, Mushagalusa K. et Kadima N : 2014. Contribution à l'étude phytochimique de quelques plantes médicinales antidiabétiques de la ville de Bukavu et ses environs (Sud-Kivu, R.D.Congo). Journal of Applied Biosciences, 75: pp 6211-6220.

N’Guessan K, Kadja B, Zirihi NG, Traoré D. et Aké-Assi L: 2009. Screening phytochimique de quelques plantes médicinales ivoiriennes utilisées en pays Krobou (Agboville, Côte d'Ivoire). Sciences et Nature, Vol. 6 N¹ : 1-15.

Nordeng H, Al-Zayadi W, Diallo D, Ballo N. et Paulsen BS : 2013. Connaissances et points de vue des praticiens de la médecine traditionnelle sur le traitement des femmes enceintes dans trois régions $\mathrm{du}$ Mali. Journal Ethnobiological Ethnomedicinal, 9: 67.

ORC Macro and the World Health Organization: 2004. Infecundity, infertility, and childlessness in 
developing countries. Demographic and Health Surveys (DHS) Comparative reports No. 9. WHO, 74 pages.

Ouattara D : 2006. Contribution à l'inventaire des plantes médicinales significatives utilisées dans la région de Divo (Sud forestier de la Côte d'Ivoire) et la diagnose du poivrier de Guinée : Xylopia aethiopica (Dunal) A. Rich. (Annonaceae). Thèse, UFR Biosciences, Université de Cocody, Abidjan, Côte d'Ivoire, $156 \mathrm{p}$.

Ouattara KA: 2007. Analyse technique et socio-économique d'une plantation de noix de cajou à Sokala Sobara, dans le Département de Dabakala. Rapport de stage de fin de première année agronomique, ESA, INP-HB. 41p.

Phillips OL, Gentry AH, Reynel C, Wilkin P. et Gálvez-Durand BC : 1994. Quantitative ethnobotany and Amazonian conservation. Conservation Biology 8: 225248.

Pourchez L: 2011. Savoirs des femmes : médecine traditionnelle et nature Maurice, Réunion, Rodrigues. Savoirs locaux et autochtones, 1. UNESCO : Paris, $120 \mathrm{p}$.

Salhi S, Fadli M, Zidane L. et Douira A : 2010. Etudes floristique et ethnobotanique des plantes médicinales de la ville de Kénitra (Maroc). Lazaroa 31: 133-146. doi:10.5209/rev_LAZA.2010.v31.9.

Thomas E, Vandebroek I, Sanca S, Van Damme P : 2009. Cultural significance of medicinal plant families and species among Quechua farmers in Apillapampa, Bolivia. Journal of Ethnopharmacology, $122: 60-67$.

Tra Bi FH, IRIÉ G M, N'gaman KCC. et Mohou CHB : 2008. Études de quelques plantes thérapeutiques utilisées dans le traitement de l'hypertension artérielle et du diabète : deux maladies émergentes en Côte d'Ivoire. Sciences \& Nature Vol.5 N¹ : 39 - 48.

Trotter R, Logan MH : 1986. Informant consensus: a newapproach for identifying potentially effective medicinal plants. In: Etkin, N.L. (Ed.), Plants in Indigenous Medicine and Diet. Redgrave Publishing Company, Bedford Hill, New York, pp. 91-112.

Yao K: 2010. Plantes médicinales et alimentaires les plus utilisées en Côte d'Ivoire: Enquêtes ethnobotaniques, recherches des activités antioxydantes. Mémoire de Diplôme d'Etudes approfondies, UFR Biosciences, Université de Cocody-Abidjan (Côte d'Ivoire), $81 \mathrm{p}$

Yapi AB : 2013. Inventaire des plantes médicinales de la famille des Asteraceae des marchés de la commune d'Abobo (Abidjan, Côte d'Ivoire). Mémoire de Master II de botanique, UFR Biosciences, Université Félix Houphouët-Boigny, 50 p.

Zerbo P, Millogo-Rasodimby J, NacoulmaOuedraogo OG. et Van Damme P : 2007. Contribution à la connaissance des plantes médicinales utilisées dans les soins infantiles en pays San, au Burkina Faso. International Journal of Biology Chemical Sciences. 1(3): 262-274

Zirihi GN: 1991. Contribution au recensement et à la connaissance de quelques espèces végétales utilisées dans la médecine traditionnelle et la pharmacopée chez les Bété du Département d'Issia, Côte d'Ivoire. Thèse de Doctorat de 3ème Cycle, Université d'Abidjan (Côte d'Ivoire), 253 p. 